\title{
Total Lung Lavage using THRIVE for Pre-oxygenation and Oxygen Insufflation During Intubation: Our Initial Experience of Two Cases
}

\author{
Vaibhavi V. Baxi ${ }^{1}$, Mansimran K. Kulaar ${ }^{1}$, Aarti Kulkarni ${ }^{1}$
}

\section{Abstract}

Introduction: Pulmonary alveolar proteinosis (PAP) is a rare disease characterized by deposition of lipoproteinaceous material in the alveoli secondary to abnormal processing of surfactant by macrophages. Total-lung lavage (TLL); a gold standard management for this disease entails washing out the proteinaceous material from the alveoli and re-establish effective oxygenation and ventilation. Transnasal humidified rapid-insufflation ventilatory exchange (THRIVE), utilizes apnoeic oxygenation to extend the apnoeic window and delay rapid de-saturation common in these cases; thus allowing for safer intubation. We would like to share our experience of two cases of TLL for PAP in which we used THRIVE for pre-oxygenation and apnoeic oxygenation during intubation. To our knowledge this is the first case report demonstrating the use of THRIVE in a case of TLL in literature.

Case presentation: We had two patients posted for TLL who were suffering from PAP; both had presented with breathlessness of increasing severity over the last few months. Both patients were young ( $35 \& 40$ yrs old) and had no other co-morbidities. Their baseline oxygen saturations $(\mathrm{SpO} 2)$ and partial pressure of oxygen in blood $(\mathrm{pO} 2)$ on air were on the lower side. Hence the decision was taken to pre-oxygenate them using THRIVE and to also use THRIVE during intubation to improve their tolerance to apnoea during intubation.

Conclusion: When used for pre-oxygenation and oxygen insufflation during intubation THRIVE prolongs the tolerable apnoea time and delays de-saturation during intubation.

Keywords: Pulmonary alveolar proteinosis (PAP); Total Lung Lavage (TLL); Transnasal humidified rapid-insufflation ventilatory exchange (THRIVE).

\section{Introduction}

Total lung lavage (TLL) is a therapeutic procedure performed for treatment of Pulmonary alveolar proteinosis (PAP), a rare disease with prevalence of approximately 1-2 per million [1]. In PAP, there is accumulation of phospholipo-proteinaceous material in the alveoli causing pulmonary gas exchange impairment [1]. A thorough TLL results in significant improvement of symptoms and radiographic appearance of lungs [1]. Lung isolation is essential for TLL and oxygenation with isolation in diseased lung is often a challenge.

Defective clearance and accumulation of surfactant in alveoli in PAP results in poor gas exchange and reduced oxygen reserve; thus significantly reducing the tolerable apnoea time in patients. We used the Transnasal humidified rapid-insufflation ventilatory exchange (THRIVE) technique of delivering up to 30 $\mathrm{L} / \mathrm{min}$ of heated and humidified air-oxygen mixture up to $100 \%$ Fraction inspired Oxygen $\left(\mathrm{FiO}_{2}\right)$ for pre-oxygenation and 70 $\mathrm{L} / \mathrm{min}$ of same for apnoeic oxygenation during intubation. This helped in maintaining oxygen saturation and gave extended apnoea duration for intubation.

In this case report we review the safety and efficacy of THRIVE for two patients undergoing TLL.

Approval of the institutional ethics committee was obtained before submitting the article for publication.

\section{Case 1}

A 35-year-old female with body mass index (BMI) $21.9 \mathrm{~kg} / \mathrm{m}^{2}$ diagnosed with PAP had an oxygen saturation $\left(\mathrm{SpO}_{2}\right)$ of $82 \%$ on room air. Serial Lung Lavage of both lungs five days apart, (left lung (with more severe disease) followed by right lung); was planned under general anaesthesia. A radial arterial line was placed prior to intubation for hemodynamic monitoring and for serial arterial blood gases (ABG). Pre-induction $A B G$ on air demonstrated a $\mathrm{pH} 7.47, \mathrm{pCO} 2-30.9$, pO2- 46 and bicarb $24.1 \mathrm{~m} \mathrm{~mol} / 1$.

Pre-oxygenation was carried out by THRIVE unit. Oxygen was administered prior to induction at $30 \mathrm{~L} / \mathrm{min}$ with an $\mathrm{FiO} 2$ of $100 \%$ till Saturation improved from $80 \%$ at room air to 97\%. Anaesthesia induction utilized fentanyl and propofol and cis-atracurium. After mask ventilation for three minutes with $1 \%$ sevoflurane on $100 \%$ oxygen, THRIVE nasal cannula was reapplied. The flow rate was

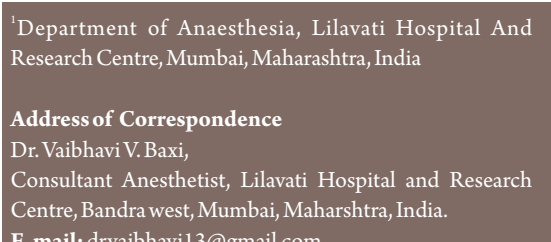

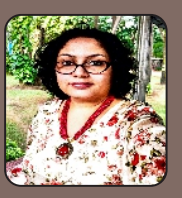

Dr. Vaibhavi V. Baxi

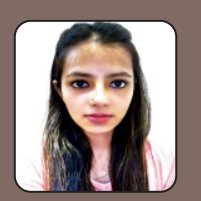

Dr. Mansimran K. Kulaar

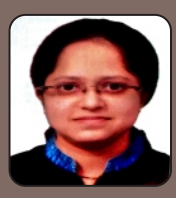

Dr. Aarti Kulkarni

(C) 2020 by Journal of Anaesthesia and Critical Care Case Reports| Available on www.jaccr.com | DOI: 10.13107/jaccr.2020.v06i03.157 This is an Open Access article distributed under the terms of the Creative Commons Attribution Non-Commercial License (http://creativecommons.org/licenses/by-nc/3.0) which permits unrestricted non-commercial use, distribution, and reproduction in any medium, provided the original work is properly cited. 
increased to $70 \mathrm{~L} / \mathrm{min}$ with an of $\mathrm{FiO}_{2} 100 \%$ during the period of intubation. Intubation was successful in the second attempt with a 35 Fr. left double lumen endotracheal tube (DLT). There was no fall in oxygen saturation during intubation. Position of DLT with adequate isolation of ventilation was confirmed with fibreoptic bronchoscope (FOB).

The right lung was ventilated with clamp on the ventilating port ofleft side of the DLT.

Anaesthesia was maintained using sevoflurane, fentanyl and cis-atracurium. We encountered high airway pressure upto 50 $\mathrm{mm}$ of $\mathrm{Hg}$ and fall in $\mathrm{SpO}_{2}$ to $70 \%$ intermittently during the procedure, which was managed by $100 \% \mathrm{FiO}_{2}$ and suction of the ventilated lung. $\mathrm{EtCO}_{2}$ went up to $48 \mathrm{~mm}$ $\mathrm{Hg}$ during the procedure. At the end of the procedure which lasted about $2.5 \mathrm{hrs}$, the DLT was exchanged to a single lumen ET. Patient was then extubated once all haemodynamic parameters and gases were within normal limits. After extubation patient was put on THRIVE again with $30 \mathrm{Lmin}^{-1}$ with $\mathrm{SpO}_{2} 95 \%$.

Postoperatively patient was shifted to ICU and her ventilation was assisted with bi-level positive airway pressure (BIPAP) overnight. With $\mathrm{FiO}_{2} 0.45$ the $\mathrm{ABG}$ showed a $\mathrm{pO}_{2}$ of 140 and $\mathrm{pCO}_{2}$ of $43 \mathrm{mmol} / \mathrm{l}$. Remarkable radiological clearance of left lung opacities along with corresponding improvement in patient's breathing pattern was seen next day.

\section{Case 2}

A 40-year-old female BMI $28.14 \mathrm{~kg} / \mathrm{m}^{2}$; diagnosed with PAP had room air $\mathrm{SpO}_{2} 89 \%$. Similar to previous case, serial lung lavage of both lungs five days apart (right lung followed by left) was planned under general anaesthesia. Pre-induction ABG with 2 $\mathrm{L} / \mathrm{min} \mathrm{O}_{2}$ via nasal prongs revealed a $\mathrm{pH}$ of 7.395, pCO2 of $40.5 \mathrm{~mm} \mathrm{Hg}, \mathrm{pO} 2$ of $96 \mathrm{~mm}$ $\mathrm{Hg}$ with bicarbonates(std) of $24 \mathrm{mmol} / 1$.

Pre-oxygenation was again conducted via THRIVE at $30 \mathrm{~L} / \mathrm{min}$ with $\mathrm{FiO}_{2} 100 \%$ until $\mathrm{SpO}_{2}$ improved from $90 \%$ to $99 \%$. After induction and mask ventilation for three minutes, the THRIVE nasal cannula was reapplied. The flow rate was increased to 70 $\mathrm{L} / \mathrm{min}$ with $\mathrm{FiO}_{2}$ of $100 \%$ during the period of intubation of trachea with a 35 Fr. DLT. We encountered airway pressures of upto $36 \mathrm{~mm}$ of $\mathrm{Hg}$ and fall in $\mathrm{SpO}_{2}$ up to $95 \%$ intermittently, which was managed by $100 \%$ $\mathrm{FiO}_{2}$. At the end of 2 hour procedure patient was extubated and put on THRIVE with 30 $\mathrm{L} / \mathrm{min}$ with $\mathrm{SpO}_{2} 100 \%$.
Postoperatively patient was shifted to ICU and her ventilation was assisted with BIPAP overnight. Significant improvement in ventilatory exchange was seen on post operative day one. For $\mathrm{FiO}_{2}$ of $40 \%$, now $\mathrm{PO}_{2}$ was $143 \mathrm{~mm} \mathrm{Hg}$ with significant clinical improvement in ease of breathing.

\section{Discussion}

PAP is characterized by the accumulation of phospholipoproteinoceous material in the alveoli that stains positive on PAS stain and its accumulation leads to compromised gas exchange [2,3]. In HRCT of the chest, reticulations superimposed with ground glass opacities form a crazy paving pattern which is characteristic of PAP $[3,4]$. The most effective palliative therapy for PAP is the mechanical removal of the proteinaceous material via TLL $[1,5-8]$.

THRIVE is the delivery of trans-nasal humidified oxygen by high-flow nasal prongs. The circuit consists of an air-oxygen blender attached to a flow meter, which controls the concentration and flow of oxygen delivered. It can generate up to $100 \% \mathrm{FiO}_{2}$ at $70 \mathrm{~L} / \mathrm{min}$, with a positive end-expiratory pressure of 7.4 $\mathrm{cm} \mathrm{H}_{2} \mathrm{O}$. Traditionally, pre-oxygenation has been performed by mask ventilation with $100 \%$ oxygen with aim to displace nitrogen in the lungs and create an oxygen reservoir. As long as a patent airway exists, the difference in rate of alveolar oxygen removal and carbon dioxide excretion generates a negative pressure gradient that pulls oxygen into the lungs. This phenomenon is known as aventilatory mass flow $[9,10]$. The concept of THRIVE revolves around extension of the apnoeic window- the small time frame following anaesthetic induction for securing a definitive airway [10]. The application of a high flow nasal delivery system provides large volumes of oxygen to the lungs and creates an oxygen reservoir, prevents entrapment of room air to ensure continual delivery of a high concentration of oxygen and reduces the rate of build-up of carbon dioxide.

Patel and Nouraei used THRIVE for preoxygenation in twenty five patients with known difficult airways, and found a mean apnoea time of $17 \mathrm{~min}$ with no desaturations below $90 \%$. Furthermore, two patients received THRIVE for the duration of their procedures, with apnoea times of 32 and 65 min [10]. Mir et al studied THRIVE in context with rapid sequence induction in e mergency surgery- THRIVE preoxygenation vs face mask preoxygenation. They concluded that THRIVE prolongs the apnoea time significantly and the blood gas profiles were similar in both groups [11]. Lodenius et al in their study of comparison of traditional face mask with THRIVE in emergency rapid sequence induction also concluded that THRIVE could have superior benefits during oxygenation as compared to a face mask [12]. Ansari et al in their study found that post operative use of high flow nasal oxygen contributed to reduced length of hospital stay in elective lung resection surgery [13].

PAP patients are prone to rapid de-saturation and de-nitrogenation alone is insufficient to counter this. THRIVE provides humidified oxygen with PEEP which prevents air entrapment and alveolar collapse.

Preoxygenation with THRIVE significantly improved the baseline $\mathrm{SpO}_{2}$ and provided us with extended apnoea time for intubation. We also utilized the THRIVE post extubation to help maintain the oxygen saturation. Patients were comfortable with the high flow of nasal oxygen. Postoperatively patients were shifted to intensive care where BIPAP was applied overnight with $40 \% \mathrm{FiO}_{2}$ as requested by the pulmonolgist as per their protocol. However there is a potential to utilise THRIVE postoperatively in intensive care as well.

\section{Conclusion}

Our study highlights the benefits of THRIVE in PAP patients by extending the apnoeic window by improving the baseline $\mathrm{SpO}_{2}$ and delaying de-saturation during intubation by continuing oxygen insufflation during the period of intubation.

\section{Clinical message}

THRIVE is a relatively newer modality of oxygen supplemetation. TLL though not a common surgery; is very demanding on the anaesthetist. Patients needing TLL often have bilateral lung disease with poor lung reserve. Lung isolation is essential in such patients and placement and confirmation of the double lumen tube is often time consuming. Use of THRIVE for preoxygenation and during intubation helps in maintaining the oxygen saturation during intubation and extends the tolerable apnoeic duration. Our experience highlights the benefits of THRIVE in PAP patients by extending the apnoeic duration by improving the baseline $\mathrm{SpO}_{2}$ by preoxygenation and delaying de-saturation during intubation by continuing oxygen insufflation during the period of intubation. We have no conflict of interests. 


\section{References}

1. Hadda V, Tiwari P, Madan K, Mohan K, Gupta N, Bharati SJ et al. Pulmonary alveolar proteinosis: Experience from a tertiary care center and systematic review of Indian literature. Lung India. 2016;33(6):626-634.

2. Awab A, Khan MS, Youness HA. Whole lung lavage - technical challenges and management of complications.J Thorac Dis. 2017;9(6):1697-1706.

3. Borie R, Danel C, Debray MP, Taille C, Dombret MC, Aubier M, Epaud R, Crestani B. Pulmonary alveolar proteinosis. European Respiratory Review. 2011 Jun 1;20(120):98-107.

4. Holbert JM, Costello P, Li W, Hoffman RM, Rogers RM. CT features of pulmonary alveolar proteinosis. AJR Am J Roentgenmaol. 2001;176(5):1287-94.

5. Shah PL, Hansell D, Lawson PR, Reid KB, Morgan C. Pulmonary alveolar proteinosis: Clinical aspects and current concepts on pathogenesis. Thorax. 2000;55:67-77.

6. Rogers RM, Levin DC, Gray BA, Moseley LW., Jr Physiologic effects of bronchopulmonary lavage in alveolar proteinosis. Am Rev Respir Dis. 1978;118(2):255-64.

7. Du Bois RM, McAllister WA, Branthwaite MA. Alveolar proteinosis: diagnosis and treatment over a 10-yearperiod. Thorax. 1983;38:360-3.
8. Kariman K, Kylstra JA, Spock A. Pulmonary alveolar proteinosis: prospective clinical experience in 23 patients for 15 years. Lung. 1984;162(4):223-31.

9. Bartlett RG Jr, Brubach HF, Specht H. Demonstration of aventilatory mass flow during ventilation and apnea in man. Journal of Applied Physiolology 1959; 14:97-101.

10. Patel A, Nouraei SA. Transnasal Humidified Rapid-InsufflationVentilatory Exchange (THRIVE): a physiological method of increasing apnoea time in patients with difficult airways. Anaesthesia. 2015;70(3):323-329.

11. Mir F, Patel A, Iqbal R, Cecconi M, Nouraei SA. A randomised controlled trial comparing THRIVE preoxygenation with facemask preoxygenation in patients undergoing rapid sequence induction of anaesthesia. Anaesthesia. 2017;72(4):439-443.

12. Lodenius A, Piehl J, Ostlund A, Ullman J, Jonsson Fagerlund M. THRIVE vs facemask breathing preoxygenation for rapid sequence induction in adults: a prospective randomised non blinded clinical trial.Anaesthesia. 2018;73(5):564-571.

13. Ansari BM, Hogan MP, Collier TJ, et al. A randomized controlled trial of high flow nasal oxygen (optiflow) as part of an Enhanced recovery after surgery (ERAS) after lung resection surgery. Ann Thoracsurg. 2016;101(2):459-464.
Conflict of Interest: Nil

Source of Support: None
How to Cite this Article

Baxi VV, Kulaar MK, Kulkarni A | Total Lung Lavage using THRIVE for Pre-oxygenation and Oxygen Insufflation During Intubation: Our Initial Experience of Two Cases | Journal of Anaesthesia and Critical Care Case Reports | September-December 2020; 6(3): 11-13. 